\title{
Monkeying around: who's learning what at the zoo?
}

\section{Courtney Keane Collins}

School of Biological, Earth and Environmental Sciences, UCC

\section{Prologue}

The following article is an account of the research I am undertaking as part of my $\mathrm{PhD}$ project. Thus far, only the preliminary research is complete, therefore final results of the project are not stated here.

\section{The school tour}

It's 9am; a fully loaded bus packed with 50 excited children leaves a school. The students' faces are already smeared with traces of chocolate, their voices are raised in excited anticipation, that familiar aroma that you can't quite describe pervades the bus; the windows are heavy with condensation. This can only mean one thing - it's that time of year again...we all remember it - THE SCHOOL TOUR, and they're going to Fota Wildlife Park. Fota Wildlife Park is one of Ireland's leading tourist attractions. Every year over 12,500 children participate in Fota's formal education programme. They come from all over Ireland to visit the park, view the animals, and have fun. We assume that it will also be an educational experience, but what, if anything, do they really learn while they are there? And how do these noisy, inquisitive, eager, groups of children influence Fota's permanent residents - the animals that live there?

Learning science outside the classroom is a powerful way to educate the public about environmental and conservation issues, and it has been shown, that not only do children benefit from informal science experiences, such as a trip to a science centre or nature park, but it is also an appealing way for them to learn. Children who come in contact with nature frequently and at an early age are thought to have more positive attitudes towards nature and conservation later in life. Zoos host millions of children at their facilities worldwide each year, and commonly claim that educating their visitors is one of their primary goals. While, there is general agreement that zoos are in a unique position to teach the public about conservation issues, their ability to successfully educate visitors has been challenged. Recently, in a British and Irish Association of Zoos and Aquariums (BIAZA) newsletter, zoos are described as being caught between 'a rock and a hard place' by Dr 
Maggie Esson ( Chester Zoo, UK) when it comes to substantiating claims they have made about their value as educators through peer-reviewed research.

\section{From awareness to action}

Some studies have shown that zoos are successful educators, especially at improving visitors' knowledge gain and possibly even their attitude toward conservation, though many of these studies have focused only on adult visitors. A recent large scale survey-based study conducted by Dr Eric Jensen (The University of Warwick, UK) and sponsored by the Zoological Society of London that did include school children, found a significant positive link between scientific learning about animals and habitats from pre to post zoo visits. There are of course many factors that influence learning in an informal setting, which have been shown to be highly personal with visitors often constructing their own meaning from a learning experience. For example, prior knowledge and experience, visitor demographics, and follow-up experiences have all been shown to affect informal science learning. Additionally, at a zoo the size and activity of the animals, the presence of zoo staff, as well as enclosure design and enrichment use may influence visitor learning.

While there is existing evidence of visitor and even children's knowledge gain as a result of a zoo trip, it has proven more difficult to establish if zoos affect any change in visitors' conservation related behaviour, which should be the ultimate learning outcome of any visit to a zoo. According to a special edition of the journal Zoo Biology which focused on zoological education, authors Ogden and Heimlich state that zoo-based education should shift its focus from 'awareness to action' from knowledge gain to behaviour change. Those studies that have assessed behaviour change as a result of a trip to a zoo have tended to focus on off-site behaviour change. For example, researchers may ask visitors questions such as 'as a result of your visit to the zoo, are you more inclined to recycle in the future or buy 'rainforest safe' products?' While a visitor, may answer 'yes', it is very difficult for a researcher to follow this up with any degree of certainty. Educational zoo research should aspire to behaviour change that is measurable, relevant, and observable on-site.

\section{The Visitor Effect}

The purpose of my research project is generally to investigate what primary school children (aged 10-12 years) learn at the zoo, while specifically studying the interactions between children and free-ranging Ring-tailed lemurs (Lemur catta), and the effect of an educational intervention on that relationship. Unique to this project, the behaviour of the children and animals will be simultaneously recorded. This will allow for both sides of the dynamic visitor-zoo animal relationship to be examined, while also considering how or if 
behaviour changes as a result of visitor education.

In the area of zoo research, the effect of visitors on animals in traditional enclosures is well documented. It is an area that is difficult to make generalisations about; and, therefore, it has generated much debate and research. Primates are by far the most well studied species. Everything from the husbandry routines at the zoo, to the 'personality' of the individual animals, to the design of the enclosure itself appears to contribute to primates' response towards visitors. Trends in the research are beginning to develop and they appear to indicate that as visitor crowds increase (particularly their noise level) primates may become more aggressive, forage less, become less social, and show more abnormal behaviours. However, very little research has been done to quantify the effect of visitors on free-ranging animals.

\section{The players}

Fota Wildlife Park has ten free-ranging ring-tailed lemurs. They are a small cat-sized primate endemic to the island of Madagascar, with a characteristic black and white striped tail. In the wild they are listed as near threatened because of habitat destruction (see Fig. 1). They are a charismatic animal that does well in captivity, and are a favourite among zoo visitors. At Fota Wildlife Park, the lemurs are restricted to the island of Fota, but they are not confined in traditional zoo enclosures. It has previously been shown that visitors learn more from animals that are free-ranging, and it also benefits the animals. However, zoo animals that are free-ranging also experience more intense interactions with visitors.

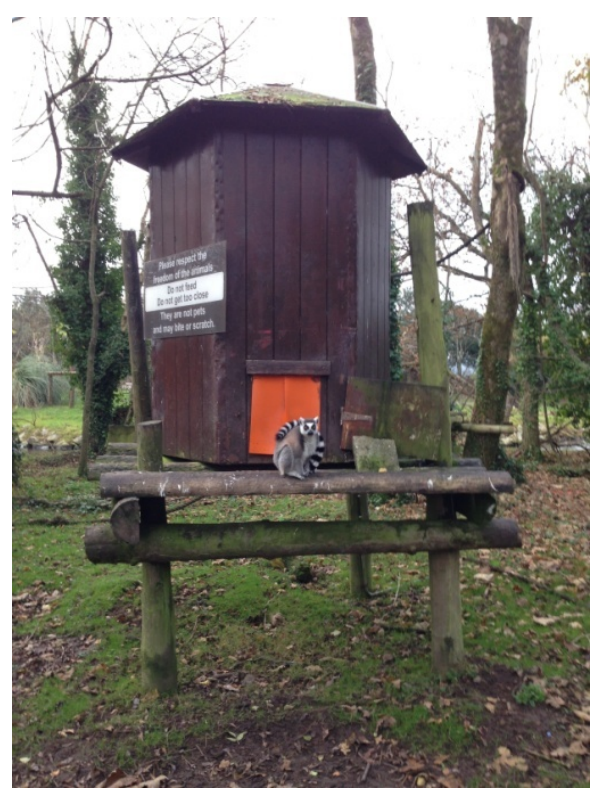

Figure 1: One of Fota's Free-range Ring-tailed Lemurs. Image: Courtney Collins. 


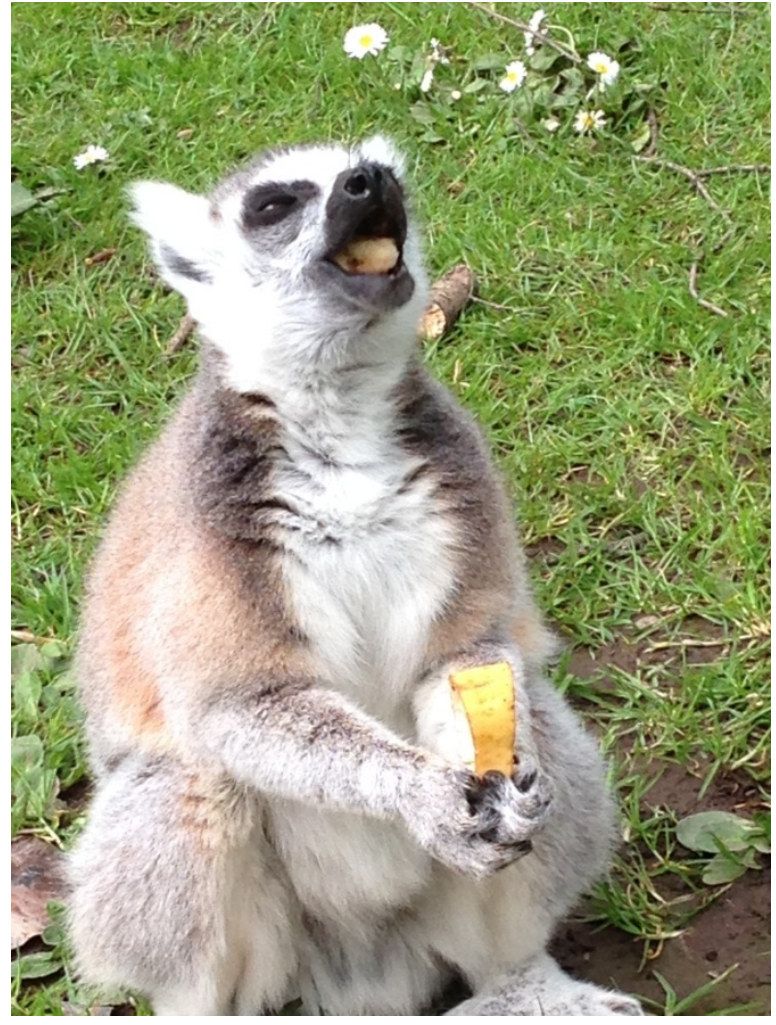

Figure 2: A Ring-tailed lemur enjoys a banana fed to it by a well-meaning visitor. Image: Courtney Collins.

\section{Can it be controlled?}

Negative visitor effects are often controlled through physical means such as barriers and sound dampening materials, but here it will be considered if education could change negative visitor behaviour and thus improve the welfare of the lemurs. It is an unfortunate fact that free-ranging animals are fed, chased, touched, and given negative attention by some visitors (especially children) at the zoo. Despite the fact that Fota Wildlife Park employs Lemur Patrol Staff to minimise the negative interactions, they still occur. Lemurs are sometimes fed ice cream, crisps, jellies and other items that are bad for their health. Figure 2 shows one of Fota's free-ranging Ring-tailed lemurs enjoying a banana fed to it by a visitor. This may seem harmless, but can quickly cause lemurs to gain too much weight and interferes with their regular diet (see Fig. 2). My research seeks to reduce negative interactions between children and lemurs through education.

\section{What are students really learning?}

Children from participating schools who visit Fota on their school tour will be given a survey before and after their visit to the park. This allows for a general assessment of their knowledge and attitude towards zoos, generally and Ring-tailed lemurs, specifically. 
During their visit to the park, children's behaviour as well as the behaviour of the Ringtailed lemurs will be observed and recorded. Some children (the treatment group) will participate in an educational intervention that relates lemurs' diet to children's diet using food pyramids; they will also have the opportunity to prepare fruit and vegetables which form part of the lemurs' daily feed. This will allow for comparison of knowledge, attitude, and behaviour of children before and after their visit to Fota, as well as between treatment and control groups. The lemurs' behaviour will also be observed, recorded, and analysed in the presence of treatment and control groups of children. Quantification of children's knowledge and attitude toward zoo animals, as well as, the interactions between children and free-ranging animals at the zoo has rarely occurred before and never in Ireland.

\section{The science behind it}

Additionally, my research will consider if there may be more to a trip to the zoo than is expected. Previously, it has been shown that many children start to lose interest in science at around the age of twelve. This has far reaching implications for future generations and is an area of increasing concern that has generated much research and interest in the last decade. There are now EU wide programmes and research specifically aimed at increasing and maintaining students' interest in science. Here, it will be considered if a school trip to Fota Wildlife Park enhances children's attitude toward science. A zoo visit is something that most children enjoy, many might even say it is their favourite place to visit, but perhaps they have never considered the science behind it? Students participating in the study will be asked questions in the pre and post surveys about their favourite subject at school, and if they like science. Additionally, during the educational intervention the fact that the zoo 'runs on science' will be emphasised and children will be challenged with questions like 'Who takes care of the animals? How does the zoo know the right food to feed them or medicine to give if animals get sick? How and why does the zoo study the animals' behaviour?' Children need continuous and sustained positive exposure to science in order to develop a positive attitude toward it, and yet, it may be equally important for children to realise that science is all around them, is part of their daily lives and that even something as enjoyable as an outing to the zoo involves science.

\section{The Impact Factor}

Given the current economic climate, and the fact that many parents may struggle to pay the fees involved in sending their children on a school tour, there is an increased expectation that children will not only have a great day out, but also learn something during a school outing. Therefore, increasing the learning potential (especially conservation behaviour change) during school visits and establishing links to science is extremely impor- 
tant for places like Fota Wildlife Park. Fota is well known for their impressive education programme and even holds the prestigious Sanford Award in education and is a designated Discover Primary Science Centre, and yet there is almost no research to support claims that learning takes place when children visit the park. Therefore, this research project is important nationally in that it will emphasise zoo-based education as a way for children to increase knowledge, and improve conservation behaviour, while learning science, and most importantly —it's fun! The research will have international implications as well since the issues addressed here pertain to zoos with education programmes worldwide. Furthermore, the research enhances existing literature about zoo-based education, and brings novel information to the area by quantifying the interactions between children and captive animals simultaneously.

I would like to thank my supervisors Dr Declan Kennedy and Dr Ruth Ramsay for their help and support with this project. Also, I would like to thank the staff at Fota Wildlife Park, Sean McKeown and Lynda McSweeney-Walsh in particular, for their support of this project. Additionally, I would like to thank the School of BEES for their financial contribution to the project. 